\title{
ENHANCING MARITAL SATISFACTION THROUGH ACCEPTANCE AND COMMITMENT THERAPY: A REVIEW OF MARITAL SATISFACTION IMPROVEMENT OF IRANIAN COUPLES
}

\author{
Nastaranalsadat Hosseini ${ }^{1}$ \\ Faculty of Education, University Malaya (UM), Malaysia. \\ (Email: nastaran62h@gmail.com) \\ Poh Li Lau ${ }^{2}$ \\ Faculty of Education, University Malaya (UM), Malaysia. \\ (Email: janicepolly@um.edu.my) \\ Diana-Lea Baranovich ${ }^{3}$ \\ Faculty of Education, University Malaya (UM), Malaysia. \\ (Email: dr.dileab@gmail.com)
}

Received date: $25-09-2019$

Revised date: 06-10-2019

Accepted date: 16-10-2019

Published date: 15-12-2019

To cite this document: Hosseini, N., Poh, L. L., \& Baranovich, D. L. (2019). Enhancing Marital Satisfaction Through Acceptance and Commitment Therapy: A Review of Marital Satisfaction Improvement of Iranian Couples. International Journal of Education, Psychology and Counseling, 4 (33), 53-60.

DOI: $10.35631 /$ IJEPC.433005

\begin{abstract}
Just about every couple considers a successful marriage a dream and aims to start spousal life, but this is an actual challenge. In reaching this aim, attaining knowledge can be a worthy initial step on the way to forming a positive and healthy marriage relationship. Acceptance and Commitment Therapy (ACT) is a new treatment with a positive impact on marital satisfaction. This form of therapy is very instrumental in bringing married couples closer and making them even more satisfied with their partners and relationships. Therefore, this paper reviews the concept of marital satisfaction and its enhancement through ACT, particularly in terms of relevance to Iranian couples. This paper additionally highlights the significance of future research on ACT for enhancing marital satisfaction among Iranian couples.
\end{abstract}

Keywords: Marital Satisfaction; Acceptance and Commitment Therapy; Marital Satisfaction Enhancement

\section{Introduction}

Historically, the organization of family and marriage has been a key factor in people's lives and the growth of nations and societies (Gladding, 2014). The family, as the most fundamental social unit, needs to be based on a strong and stable marital relationship. This relationship is 
linked to marital satisfaction, which leads to a healthy marriage. In contrast, marital dissatisfaction may result in divorce and lasting separation. Although the rate of divorce has reduced from the highest by roughly 1980, it is again high worldwide. These days, marital dissatisfaction and divorce have become a joint incident worldwide (Galvin, Braithwaite, \& Bylund, 2015; Gladding, 2014). As studies reveal, the divorce rate has recently been on considerable rise in Iran (Aghajanian \& Thompson, 2013; Mohammadi \& Tafti, 2014). Consistent with official statistics from the Statistical Centre of Iran, the divorce rate increased from $10.6 \%$ to $18.6 \%$ compared to the marriage rate through the same period from 2005 to 2012 (Statistical Center of Iran, 2014). Reasonable treatment can prevent the rising divorce rate issue and help Iranian couples possess more diverse skills and capabilities to control situations than earlier. ACT is a novel, academic method of studying marital satisfaction and marital relationships (Harris, 2010) that has recently been receiving attention in Iran and is applied for Iranian couples.

\section{Marital Satisfaction}

The essential aim of marriage is satisfaction. In fact, satisfaction with marriage stands as an overall estimation of one's statement of marriage and as an image of a content and effective marriage (Boerner, Jopp, Carr, Sosinsky, \& Kim, 2014), whereby couples seek the highest levels of satisfaction from their marital life. Social researchers deem marital satisfaction one of the initial concepts in studying marital relationships (Lewis \& Spanier, 1979) and an essential element for generating good couple relationships (Zakhirehdari, Navabinejad, \& Koraei, 2019).

Numerous factors and elements affect the multidimensional and broad notion of marital satisfaction. Provided the factors are fulfilled, marital satisfaction is a success (Fincham, Beach, \& Davila, 2004; Laurenceau, Barrett, \& Rovine, 2005; Vangelisti \& Huston, 1994). In fact, there is a link between these factors and marital satisfaction, whereby higher factor levels or the partial connections to their similarity levels lead to satisfaction with marriage (Steinmetz, Clavan, \& Stein, 1990). Included are psychological factors like temper and individual character, spousal communication (Bradbury, Fincham, \& Beach, 2000; P. Greeff, 2000), sexual relationship and interaction (Rosen-Grandon, Myers, \& Hattie, 2004), as well as sociodemographic factors like educational level, age, income (Conger, Rueter, \& Elder Jr, 1999), religion, workload and even the transition to parenthood (Slavinskienė \& ŽardeckaitèMatulaitienè, 2012; Waite \& Lillard, 1991).

Researchers are continuously investigating the role of gender differences on marital satisfaction (Jain, 2014; Kurdek, 1995; Shen, 2002; Vangelisti \& Huston, 1994; Zakhirehdari et al., 2019), because it is described through differences in gender role too (Bernard, 1972; Fowers, 1991; Mickelson, Claffey, \& Williams, 2006). Gender roles comprise the behaviours, attitudes and values that a particular culture considers appropriate for males and females. Today, through shifting gender role attitudes toward egalitarianism, principally among educated couples, openness has increased and consequently led to the emergence of structural gender equality. Couples therefore experience more equal roles and opportunities in any aspect of marital life and can thus live and know greater satisfaction. However, unfulfilled aforementioned factors precede marital conflicts between couples. Such complex discordant interactions may have not only a damaging impact on the individual's mentality and personality but also on the marriage (Benson, McGinn, \& Christensen, 2012).

Some techniques and treatments are practical in decreasing several types of such difficulties and consequently improving marriage relationships along with satisfaction. Novel 
investigations into marital satisfaction (Dewane, 2008) now also involve the new approach of Acceptance and Commitment Therapy (ACT).

\section{Acceptance and Commitment Therapy}

Acceptance and Commitment Therapy, or ACT, is known as the third wave of behaviour therapy established by Steven C. Hayes in the late 1980s. The assumption shared by the first two waves of Behavioural Therapy (BT) is that specific emotions, cognitions and physiological conditions produce dysfunctional behaviour; consequently, therapeutic intervention is designed to remove or at least decrease these problematic internal events. The objectives of thirdgeneration therapies are shifting from purely decreasing symptoms to boosting abilities expected to meaningfully improve the quantity and quality of actions in which the patient would find value (Hayes, 2004).

ACT displays no focus on symptom reduction as a target, in complete contrast to the majority of western psychotherapy. The base of this view is that continuous effort to remove the symptoms essentially makes a clinical disorder in the first place. Considering a specific experience, a symptom generates conflict with the symptom. Upon hearing something labelled as a symptom, the ensuing attempt is to remove the label due to the associated accompanying term "pathological." However, the goal of ACT is to transform one's relationship with their problematic thoughts to consequently not perceive them as symptoms any longer. As an alternative, one absorbs and observes these thoughts as harmless, albeit uncomfortable, passing psychological events. Actually, through this process ACT essentially reduces the symptom, but rather as a side effect and not the aim.

The purpose of ACT is in fact to improve psychological flexibility over the six subsequent stages of psychological change: acceptance, defusion, being present, self as context, values, and behavioural commitments (Hayes, Luoma, Bond, Masuda, \& Lillis, 2006).

Based on the work of psychologist Steven Hayes, ACT encourages individuals to recognize what they are not able to control, to control anything they are capable of controlling and learn to acknowledge the difference between what they can and cannot control. ACT teaches to agree and accept even undesirable thoughts. Thus, with ACT, individuals acquire skills to control their behaviour and subsequently make their life meaningful and consistent with their sincere beliefs. Hayes does not accept the "control agenda" that leads individuals to feel they can face the signs that render them abnormal or sick if only they could control their inner feelings and thoughts. Rather, Hayes considers an acceptable treatment as starting with accepting undesirable feelings and thoughts as normal. Hence, ACT helps clients feel better and have a more valued life. In particular, through facing an extensive range of typical emotions, clients learn to embrace negative feelings, thoughts and memories, manage the present discomfort and anxiety or pain that evidently come with living a full life(Forman \& Herbert, 2009) and choose directional values.

\section{Acceptance and Commitment Therapy for Couples Who have Marital Problems}

From an ACT perspective, rigid, inflexible and impractical control and strategies such as experiential avoidance that the relationships of couples contextualize can result in the development of problems that will continue to persist, including emotional distance, distress and conflict (Harris, 2010). A common instance of such strategy is where the partners avoid communication since a previous encounter resulted in conflict and emotional distance. Another instance is partners avoiding expressing their emotions or avoiding physical intimacy because 
they fear rejection. Hence, they may avert activities that are part of creating shared memories and meaning (Baruch, Kanker, \& Busch, 2012). These experiential avoidance strategies support both partners to avoid unwanted feelings, talks and thoughts, which at the same time affects the couple's actions and can thus be unconstructive to their relational health.

Moreover, a relationship may involve entanglements and compromising situations that carry feelings and thoughts about prior actions or lack thereof. Holding on to, and acting on the respective thoughts can maintain negative cycles in a couple's relationship (Peterson, Eifert, Feingold, \& Davidson, 2009).

ACT serves to weaken such processes and reduce the unnecessary suffering that experiential avoidance causes in the partners. One of the ultimate goals of ACT is to help each partner become attentive to his/her emotional and cognitive responses (Baruch et al., 2012) and to observe his/her behaviour in the relationship (Harris, 2010). This assists couples clarify their shared relationship values and commit to acting toward these values even when encountering undesirable thoughts, actions and feelings.

Essentially, the difference between previous learning techniques and ACT is that the prior teaches couples to avoid the kinds of situations that will hurt or result in conflict or rejection. The latter helps couples approach unpleasant feelings, acts, thoughts and bodily states related or linked to such adverse relationship dynamics and patterns. The partners can then easily observe a situation at the time when one is hurt emotionally or feels invalidated, which would otherwise result in emotional distancing. Emotional distancing protects a couple from possible further distress, but ACT teaches the couple to defuse the feelings and thoughts that may lead to emotional distancing. They learn to mindfully accept such thoughts and act toward consistence and shared relationship values regarding their connection and emotional intimacy. When a couple begins to utilise these skills and strategies, the partners will become more able and willing to deal with a situation they had avoided before. The partners will thus benefit from a new opportunity to behave in a way that may yield profounder satisfaction with their relationship and their interpersonal intimacy may increase over time as well. ACT does not guarantee that the situation will improve; but because it targets a couple's experiential avoidance in the context of their own relationship, ACT helps the partners approach previous unwanted thoughts constructively toward their shared values and provides the chance to create a stronger connection (Peterson et al., 2009).

ACT encourages partners to learn to recognize their own (positive or likely negative) interpretations of their partner's behaviour as thoughts that require no action, after which they accept their own internal reactions mindfully. ACT employs 'cognitive defusion techniques' specifically to help partners decrease the believability of their own thoughts, therefore reducing the individual's tendency to "become" his/her thoughts. The result is greater flexibility and an emerging space for new relationship behaviours.

Moreover, ACT uses value-directed actions to expand on strategies for behaviour change. It promotes changes in behaviour by elucidating the individual's values and those shared in the relationship. As such, the partners will commit to acting toward their shared values by linking them to long- and short-term goals (Harris, 2010). Clients thus receive the support to practice the acceptance and willingness of behaviours even in the presence of negative internal interpretations and evaluations. ACT applies interventions with an experiential basis to undermine and weaken the verbal rules in a relation that govern the individuals' behaviour. For 
this purpose, ACT makes use of metaphors, mindfulness techniques and other experiential exercises. Clients learn to respond less literally to their own emotions and thoughts, to remain mindful and observant, and to not act on feelings and thoughts especially in the context of the relationship.

Cognitive defusion, moving toward the valued directions in relationships and mindful acceptance as the core interventions in ACT help partners start committing to behaviours that would hopefully result in higher satisfaction in the relationship and lower interpersonal distress (Peterson et al., 2009).

\section{ACT and Marital Satisfaction Improvement of Iranian Couples}

Previous research studies done on ACT and marital satisfaction improvement among Iranian couples present a clear picture of the enhanced marital satisfaction achieved through Acceptance and Commitment Therapy.

Moradzadeh and Pirkhaefi (2018) determined The Effectiveness of Acceptance and Commitment Therapy on Marital Satisfaction and Cognitive Flexibility among Married Employees of the Welfare Office. The research was semi-experimental with a pre-test and a post-test. The statistical population included all married employees of the welfare office in Varamin city in 2016. The availability sampling method generated 30 individuals who were randomly assigned to 2 equal groups. The experimental group underwent 8 sessions of 90 minutes. Both groups completed Olson and Flowers' questionnaire on marital satisfaction as a pre-test and Dennis and Vander Wal's cognitive flexibility questionnaire as a post-test. The data underwent the multivariate and univariate analysis of covariance. The findings showed a significant difference between the experimental and control groups in two variables: marital satisfaction and cognitive flexibility. In other words, ACT led to a significant increase in the marital satisfaction and cognitive flexibility of married employees.

Similarly, Kavousian, Harifi, and Karimi (2017) studied the efficacy of Acceptance and Commitment Therapy (ACT) on the marital satisfaction of married couples. They used a quasiexperimental pretest-posttest study design. The population consisted of all students at the Islamic Azad University of Bandar Abbas who were married during 2015 and 2016. Based on the acceptance and commitment therapy protocol the researchers conducted a 12-session intervention over a period of 3 months and used the Enrich marital satisfaction assessment questionnaire to collect data. The subjects responded to the questionnaire before the intervention and 1 month after the intervention. Descriptive statistics and ANOVA were used for data analysis in SPSS V.20. According to the results, the difference in the average post-test scores between the two groups was statistically significant for the following marital satisfaction components: sexual satisfaction (experimental, 24.45; control, 19.83), communication (experimental, 22.10; control, 19.81), solving conflict (experimental, 20.35; control, 17.15) and idealistic distortion (experimental, 16.65; control, 14.55). Based on the findings, the researchers recommended using ACT alongside other interventions for improving the marital satisfaction of couples.

Later, Omidi and Talighi (2017) investigated the efficacy of marital therapy based on Acceptance and Commitment Therapy (ACT) on marital satisfaction and quality of life (QOL) through a semi-experimental study. They divided a sample of 50 couples from the Zehn Agah Clinic at Bonyad-e Shahid Centre and the Counselling Centre of Kargarnezhad Hospital in Kashan in 2015 into two groups for ACT and treatment as usual. ENRICH was the study 
instrument employed. The experimental group received ACT for 10 weeks (90min sessions) in group therapy for couples and the control group received no treatment. The results demonstrated a significant difference in the mean scores of marital satisfactions and QOL between the two groups, meaning the Acceptance and Commitment Therapy enhanced the marital satisfaction and QOL of couples. The researchers thus found that ACT for couples was an effective intervention in treating clients with marital problems.

Keyvanpour and Kashani (2014) studied the influence of ACT on the increase in the rate of marital satisfaction among married women in Iran. They separated a sample of 30 participants from Shams Abad-Majidieh district into two groups of 15. The researchers assessed the participants prior to and after the intermediation with the ENRICH marital satisfaction questionnaire (a 47-question form). The covariance analysis outcomes specified that this therapy approach was effective for marital satisfaction.

The above-cited study showed that emphasis on the role of marital satisfaction development is ultimately beneficial to boosting life quality. In addition, based on the experimental group's feedback, the ACT treatment method appeared to significantly affect marital life. One explanation for the positive effect is the formation and growth of acceptance rather than treatment changes. Another interpretation is the influence of the radical but essential attention in this treatment on people's living nature. With this approach and regardless of other processes, instead of forcing a lifestyle upon clients, they were free to select a lifestyle based on their own values. Iran as a developing country is undergoing a conversion from traditionalism to modernism that is inconsistent with the confusion linked to this alteration.

Referring to the findings, it is probable that ACT intervention strategies are practical for a wide range of matters and particularly for married couples. By considering ACT as an experienceconstructing treatment, therapists should anticipate transferring specific concepts, behaviours and thoughts via training to direct clients toward a straight experience. For instance, participants reported being able to cope with difficulties by using metaphors in numerous challenging circumstances.

Furthermore, Arabnejad, Birashk, Abolmaali and Alhosseini (2014) determined the effectiveness of Acceptance and Commitment Therapy (ACT) on the reduction of marital conflict. They chose a sample of 30 couples randomly for the 8-session ACT protocol and analysed the data in MANCOVA. The results presented a significant difference between the test groups in terms of the marital conflict variable, thereby validating the hypothesis concerning the effectiveness of ACT on reducing couples' marital conflict. Moreover, the findings of this research are in line with other studies, e.g. Baroch, Kanker \& Bosij (2009), Hafman \& Smondes (2008) and Broto et al. (2007).

This section provided a summary of literature related to marital satisfaction enhancement based on Acceptance and Commitment Therapy (ACT) among Iranian couples in particular. Overall, the literature suggests that ACT increases the marital satisfaction of Iranian couples.

\section{Conclusion}

In conclusion, Acceptance and Commitment Therapy (ACT) is an affective intervention in the treatment of couples with marital problems. The success of ACT in enhancing marital satisfaction gives credence to the Hayes model as a viable marital relationship development process model. ACT can help enhance marital satisfaction only when both partners approach 
their own unpleasant feelings, actions, thoughts and bodily states that are linked to the relationship dynamics. ACT is also effective when the couple can mindfully accept such thoughts and actions toward the consistence and shared values of the relationship regarding their connection and emotional intimacy. Future research efforts may present more impacts of ACT on marital satisfaction among Iranian couples as well as contribute to the marriage and family counselling field.

\section{References}

Aghajanian, A., \& Thompson, V. (2013). Recent divorce trend in Iran. Journal of Divorce \& Remarriage, 54(2), 112-125.

Baruch, D., Kanker, J., \& Busch, A. (2012). Acceptance and commitment therapy: enhancing the relationships. Journal of clinical case studies, 8(3), 241-257.

Benson, L. A., McGinn, M. M., \& Christensen, A. (2012). Common principles of couple therapy. Behavior therapy, 43(1), 25-35.

Bernard, J. (1972). The future of marriage. New York: Bantam. Carr D.(2004). The Desire to Date and Remarry Among Older Widows and Widowers. J. Marriage Fam, 66, 10511068.

Boerner, K., Jopp, D. S., Carr, D., Sosinsky, L., \& Kim, S.-K. (2014). "His" and "her" marriage? The role of positive and negative marital characteristics in global marital satisfaction among older adults. Journals of Gerontology Series B: Psychological Sciences and Social Sciences, 69(4), 579-589.

Bradbury, T. N., Fincham, F. D., \& Beach, S. R. (2000). Research on the nature and determinants of marital satisfaction: A decade in review. Journal of marriage and family, 62(4), 964-980.

Conger, R. D., Rueter, M. A., \& Elder Jr, G. H. (1999). Couple resilience to economic pressure. Journal of personality and social psychology, 76(1), 54.

Dewane, C. (2008). The ABCs of ACT: Acceptance and commitment therapy. Social Work Today, 8(5), 34 .

Fincham, F. D., Beach, S. R., \& Davila, J. (2004). Forgiveness and conflict resolution in marriage. Journal of family Psychology, 18(1), 72.

Forman, E. M., \& Herbert, J. D. (2009). New directions in cognitive behavior therapy: Acceptance-based therapies. General principles and empirically supported techniques of cognitive behavior therapy, 77-101.

Fowers, B. J. (1991). His and her marriage: A multivariate study of gender and marital satisfaction. Sex Roles, 24(3-4), 209-221.

Galvin, K. M., Braithwaite, D. O., \& Bylund, C. L. (2015). Family communication: Cohesion and change: Routledge.

Gladding, S. T. (2014). Family therapy: History, theory, and practice: Pearson Higher Ed.

Harris, R. (2010). ACT with love: Stop struggling, reconcile differences, and strengthen your relationship with acceptance and commitment therapy: ReadHowYouWant. com.

Hayes, S. C. (2004). Acceptance and commitment therapy, relational frame theory, and the third wave of behavioral and cognitive therapies. Behavior therapy, 35(4), 639-665.

Hayes, S. C., Luoma, J. B., Bond, F. W., Masuda, A., \& Lillis, J. (2006). Acceptance and commitment therapy: Model, processes and outcomes. Behaviour research and therapy, 44(1), 1-25.

Jain, A. (2014). Gender role attitudes and marital satisfaction among Asian Indian couples living in the US: an exploratory study. Rutgers University-Graduate School of Applied and Professional Psychology. 
Kurdek, L. A. (1995). Predicting change in marital satisfaction from husbands' and wives' conflict resolution styles. Journal of Marriage and the Family, 153-164.

Laurenceau, J.-P., Barrett, L. F., \& Rovine, M. J. (2005). The interpersonal process model of intimacy in marriage: A daily-diary and multilevel modeling approach. Journal of family psychology, 19(2), 314.

Lewis, R. A., \& Spanier, G. B. (1979). Theorizing about the quality and stability of marriage. Contemporary theories about the family: research-based theories/edited by Wesley R. Burr...[et al.].

Mickelson, K. D., Claffey, S. T., \& Williams, S. L. (2006). The moderating role of gender and gender role attitudes on the link between spousal support and marital quality. Sex Roles, 55(1-2), 73-82.

Mohammadi, S. M. R. G., \& Tafti, F. C. (2014). Socioeconomic factors affecting divorce in Islamic Republic of Iran (Yazd). International Journal of Business and Social Science, $5(5)$.

P. Greeff, T. D. B., Abraham. (2000). Conflict management style and marital satisfaction. Journal of Sex \& Marital Therapy, 26(4), 321-334.

Peterson, B. D., Eifert, G. H., Feingold, T., \& Davidson, S. (2009). Using acceptance and commitment therapy to treat distressed couples: A case study with two couples. Cognitive and Behavioral Practice, 16(4), 430-442.

Rosen-Grandon, J. R., Myers, J. E., \& Hattie, J. A. (2004). The relationship between marital characteristics, marital interaction processes, and marital satisfaction. Journal of Counseling \& Development, 82(1), 58-68.

Shen, A. C.-T. (2002). Developing a multi-dimensional marital satisfaction inventory for Taiwanese couples. Formosa Journal of Mental Health, 15(3), 67-100.

Slavinskienè, J., \& Žardeckaitè-Matulaitienė, K. (2012). The relations between sociodemographical factors, irrational beliefs, adult insecure attachment style and marital satisfaction of Lithuanian married couples. Proceedings in ARSA-Advanced Research in Scientific Areas (1).

Steinmetz, S. K., Clavan, S., \& Stein, K. F. (1990). Marriage and family realities: Historical and contemporary perspectives: Harper and Row.

Vangelisti, A. L., \& Huston, T. L. (1994). Maintaining marital satisfaction and love.

Waite, L. J., \& Lillard, L. A. (1991). Children and marital disruption. American journal of sociology, 96(4), 930-953.

Zakhirehdari, N., Navabinejad, S., \& Koraei, A. (2019). The effectiveness of cognitivebehavioral couple therapy on improving marriage performance and marital intimacy of couples. 